\title{
Improving coping mechanisms of Malaysian adolescents living in orphanages through a life skills education program: a multicentre randomized controlled trial
}

\begin{abstract}
The current study amid to determine whether a life skills-based education could improve coping skills among adolescents in Malaysian orphanages. It was a randomized controlled trial comprising intervention and control groups which were randomly selected to receive the life skills, or the Placebo education programmes. The DASS21 and Brief COPE were used as the study instruments. Results showed the mean scores of self-distraction, active coping, use of emotional support, use of instrumental support, positive reinterpretation, planning and acceptance, at post-test, were significantly increased compared to the pre-test values. Denial, substance abuse, behavioural disengagement and self-blame significantly decreased. The findings provide an opportunity to assess the effects of participation in a life skills education programme on behavioural health in Malaysia orphanages.
\end{abstract}

Keyword: Adolescents behavioural health; Life skills education; Emotional problems; Coping mechanisms; Malaysian orphanages 